OPEN ACCESS

Edited by:

Fabrice Cognasse,

Groupe Sur L'immunité Des Muqueuses Et Agents Pathogènes

(GIMAP), France

Reviewed by:

Martin Metz,

Charité - Universitätsmedizin

Berlin, Germany

Arifumi Iwata,

Chiba University, Japan

*Correspondence: Bradford A. Youngblood byoungblood@allakos.com

Specialty section: This article was submitted to

Inflammation,

a section of the journal

Frontiers in Immunology

Received: 06 January 2021 Accepted: 18 February 2021

Published: 10 March 2021

Citation:

Gebremeskel S, Schanin J, Coyle KM, Butuci M, LuU T, Brock EC, Xu A

Wong A, Leung J, Korver W, Morin RD, Schleimer RP, Bochner BS and Youngblood BA (2021) Mast Cell and Eosinophil Activation Are Associated With COVID-19 and TLR-Mediated Viral Inflammation: Implications for an Anti-Siglec-8 Antibody. Front. Immunol. 12:650331. doi: 10.3389/fimmu.2021.650331

\section{Mast Cell and Eosinophil Activation Are Associated With COVID-19 and TLR-Mediated Viral Inflammation: Implications for an Anti-Siglec-8 Antibody}

\author{
Simon Gebremeskel ${ }^{1}$, Julia Schanin ${ }^{1}$, Krysta M. Coyle ${ }^{2}$, Melina Butuci ${ }^{1}$, Thuy Luu ${ }^{1}$, \\ Emily C. Brock ${ }^{1}$, Alan Xu ${ }^{1}$, Alan Wong ${ }^{1}$, John Leung ${ }^{1}$, Wouter Korver ${ }^{1}$, Ryan D. Morin ${ }^{2}$, \\ Robert P. Schleimer ${ }^{3}$, Bruce S. Bochner ${ }^{3}$ and Bradford A. Youngblood ${ }^{1 *}$

\begin{abstract}
${ }^{1}$ Allakos Inc., Redwood City, CA, United States, ${ }^{2}$ Department of Molecular Biology and Biochemistry, Research Centre, Simon Fraser University, Vancouver, BC, Canada, ${ }^{3}$ Division of Allergy and Immunology, Department of Medicine, Northwestern University Feinberg School of Medicine, Chicago, IL, United States
\end{abstract}

Coronavirus disease 2019 (COVID-19) caused by SARS-CoV-2 infection represents a global health crisis. Immune cell activation via pattern recognition receptors has been implicated as a driver of the hyperinflammatory response seen in COVID-19. However, our understanding of the specific immune responses to SARS-CoV-2 remains limited. Mast cells (MCs) and eosinophils are innate immune cells that play pathogenic roles in many inflammatory responses. Here we report MC-derived proteases and eosinophil-associated mediators are elevated in COVID-19 patient sera and lung tissues. Stimulation of viral-sensing toll-like receptors in vitro and administration of synthetic viral RNA in vivo induced features of hyperinflammation, including cytokine elevation, immune cell airway infiltration, and MC-protease production-effects suppressed by an anti-Siglec-8 monoclonal antibody which selectively inhibits MCs and depletes eosinophils. Similarly, anti-Siglec-8 treatment reduced disease severity and airway inflammation in a respiratory viral infection model. These results suggest that $\mathrm{MC}$ and eosinophil activation are associated with COVID-19 inflammation and anti-Siglec-8 antibodies are a potential therapeutic approach for attenuating excessive inflammation during viral infections.

Keywords: COVID-19, SARS-CoV-2, Toll-like receptor, mast cell, eosinophil, Siglec-8, lirentelimab, viral inflammation

\section{INTRODUCTION}

The rapid spread of severe respiratory syndrome coronavirus 2 (SARS-CoV-2) and resulting coronavirus disease 2019 (COVID-19) pose an unprecedented global health crisis. While the majority of cases resolve with mild symptoms or no symptoms at all, some patients develop fatal complications, such as acute respiratory distress syndrome (ARDS), for which effective therapeutic strategies are urgently needed $(1,2)$. 
In these severe cases, a hyperinflammatory response or cytokine storm has been observed and is suspected to be a potential driver of pathology $(3,4)$. Indeed, transcriptomic profiling and histologic examination of the lungs or bronchoalveolar lavage (BAL) fluid of COVID-19 patients have revealed extensive immune cell infiltration and significantly elevated levels of cytokines, chemokines, and other proinflammatory mediators that correlate with disease severity (5-7). Evidence currently points to immune dysfunction as a potential driver of these hallmark characteristics of COVID-19. However, our understanding of the specific immune responses to SARS-CoV-2 remains extremely limited.

Innate immune sensing serves as the first line of antiviral defense and is initiated by the recognition of conserved pathogen-associated molecular patterns by pattern recognition receptors (PRRs). Single-stranded RNA (ssRNA) viruses, such as SARS-CoV-2, replicate via formation of double-stranded RNA (dsRNA) intermediates, which can be detected by Toll-like receptor (TLR) 3 and cytosolic PRRs MDA-5 and RIG-1, while ssRNA can be detected by TLR7 and TLR8 (8). Indeed, activation of immune cells via PRRs has been postulated to drive the release of proinflammatory cytokines seen in severe COVID-19 patients $(8-10)$.

Mast cells (MCs) are tissue resident immune cells that constitute a major sensory arm of the innate immune system. They are crucially located at sites that interface with the external environment, such as the lungs and gastrointestinal tract, allowing them to be among the first cells to respond during pathogen invasion (11). MCs are equipped with TLRs and receptors for inflammatory mediators, allowing them to act as sentinels for tissue damage and pathogen exposure (12). Upon activation, MCs release preformed granules containing inflammatory mediators, vasoactive autocoids, and catalytically active MC-specific proteases, including $\beta$-tryptase, chymase, and carboxypeptidase (CPA)-3 (13). In humans, MCs are classified according to their protease content and tissue distribution, with the $\mathrm{MC}_{\mathrm{T}}$ subclass expressing only tryptase and being primarily found in mucosal tissues and the $\mathrm{MC}_{\mathrm{TC}}$ subclass expressing tryptase, chymase, and CPA-3 and located mainly in the skin (13). MC activation also leads to de novo production of cytokines and lipid mediators, including TNF, IL-6, CCL2, CCL3, prostaglandin D2 and E2, and leukotriene B4 and C4 $(14,15)$, many of which are now known to be associated with the cytokine storm observed in COVID-19 $(5-7,16)$.

$\mathrm{MC}$ responses to viral pathogens have not been extensively studied. Viruses can activate MCs directly or indirectly through viral or inflammatory products such as, ssRNA or dsRNA replication intermediates, complement, and cytokines (17). Many viruses have been shown to induce MC degranulation, protease release, and cytokine production, including dengue (DENV), respiratory syncytial virus (RSV), herpes simplex virus (HSV), Japanese encephalitis (JEV), Zika, and influenza $(18,19)$. The interactions between MCs and viruses or pathogen-derived products are complex and can result in either beneficial or detrimental outcomes $(17,18)$. For example, MCs have been shown to play a protective role against HSV and vaccinia virus infection $(17,19)$. In contrast, tryptase and chymase are elevated in plasma from patients with severe DENV infection and these $\mathrm{MC}$ proteases were shown to induce significant vascular leakage in peripheral tissues in response to the infection (20).

Sialic acid-binding immunoglobulin-like lectin (Siglec)-8 is an inhibitory receptor, selectively expressed on MCs and eosinophils, that inhibits MC activation and induces eosinophil death and depletion when engaged with a monoclonal antibody (mAb) (21-23). Anti-Siglec-8 mAbs have been shown to suppress immune cell infiltration, local and systemic inflammation, protease production, fibrosis, and anaphylaxis $(24,25)$. Clinical evaluation of lirentelimab (AK002), a humanized anti-Siglec-8 $\mathrm{mAb}$, is currently underway in multiple mast cell and eosinophildriven diseases $(26,27)$.

Given the pathogenic role of MCs in many inflammatory diseases and their putative role in COVID-19 pathogenesis (28-30), we sought to evaluate MC activation in SARS-CoV-2 patients and the activity of a Siglec- $8 \mathrm{mAb}$ in models of viral inflammation using Siglec- 8 transgenic mice. Here we show that MC-derived proteases are significantly elevated in COVID-19 patient sera and lung autopsies. Surprisingly, we also found evidence of eosinophil activation in these COVID-19 patients. Stimulation of viral-sensing toll-like receptors in vitro and administration of synthetic viral RNA in vivo induced local and systemic inflammation, including cytokine elevation, immune cell airway infiltration, MC-protease production, and eosinophil-granule release. Treatment of Siglec- 8 transgenic mice with an anti-Siglec-8 $\mathrm{mAb}$ significantly suppressed airway inflammation induced by either administration of synthetic viral RNA or infection with RSV. These data provide evidence that MC activation is a component of COVID-19 inflammation and demonstrate that targeting Siglec-8 with a mAb suppresses TLR- and RSV-mediated inflammation, supporting anti-Siglec- 8 antibodies as a potential therapeutic approach for attenuating excessive inflammation during viral infections.

\section{MATERIALS AND METHODS}

\section{Human Serum Samples}

Sera from uninfected controls $(n=20)$ and SARS-CoV-2 patients $(n=19)$ were obtained from Discovery Life Sciences Biobank (San Luis Obispo, CA, USA). The SARS-CoV-2 status of these donors was determined using the Abbott RT-PCR nasopharyngeal swab test. Serum cytokine levels were quantified using multiplex analysis Meso Scale Discovery (MSD). Mast cell activation was quantified in serum using MC-derived proteases: $\beta$-tryptase (ELH-TPSB2-1, Ray Biotech), CPA3 (CPA3, LS-F7363, LS Bio) or chymase (50-149-8059, Biomatik). Mast cell tryptase activity (mature tryptase) was determined by the Tosyl-Gly-ProLys-pNA-based method (IMM001, Sigma-Aldrich) according to the manufacture's instructions. Human eosinophil-derived neurotoxin (EDN) was measured by ELISA (LS-F12507, LS Bio).

\section{Mice and Models of Viral Inflammation}

Siglec-8 tg mice were generated as previously described (25) Siglec- 8 tg mice were injected with an anti-Siglec- 8 mIgG1 mAB (2E2 clone, Allakos, Inc) or isotype-matched control mIgG1 
$\mathrm{mAb}$ (Biolegend) intraperitoneally at $5 \mathrm{mg} / \mathrm{kg} 3 \mathrm{~h}$ before poly (I:C) challenge (Invivogen). On days 1 and 2, the mice were anesthetized by isoflurane inhalation and $50 \mathrm{ul}$ of PBS or poly (I:C) (1 mg/ml) was administered intratracheally. On day 3, serum, bronchoalveolar lavage (BAL) fluid, lungs and peripheral blood were isolated. Cytokines mice were measured using the multiplex Meso Scale Diagnostics (MSD) assay. For the RSV infection model, Siglec- 8 tg mice were were injected with an anti-Siglec- 8 mIgG1 mAB (2E2 clone, Allakos, Inc.) or isotypematched control mIgG1 mAb (Biolegend) intraperitoneally at $5 \mathrm{mg} / \mathrm{kg} 4 \mathrm{~h}$ before being anesthetized with isoflurane and infected with $1.7 \times 10^{6} \mathrm{pfu}$ RSVA2 in $100 \mu \mathrm{l}$ via the intranasal route. Following infection, disease severity was assessed daily by measuring body weight. On day 7 the study was terminated, and BAL fluid was collected for differential counts and viral infectivity assay. RSV A2, an A subtype RSV, was obtained from ATCC and prepared by infecting semiconfluent layers of HEp2 cells. When the infected monolayers exhibited approximately $80 \%$ confluence, the media was clarified and supernatant was snap frozen on dry ice.

Additional methods are described in the Supplementary Material.

\section{RESULTS}

\section{MC-Specific Proteases Are Significantly Elevated in SARS-CoV-2 Patient Serum}

Previous studies have demonstrated that activation of MCs and subsequent protease release contribute to virus-induced inflammation and pathology, including vascular leak, excessive airway inflammation, barrier disruption, and fibrosis (20,31). As such, many reports have implicated MCs as putative effector cells in COVID-19 pathogenesis, however, to date, no studies have directly examined the role of MCs (32-35). To evaluate if MC activation was associated with SARS-CoV-2 inflammation, we tested the serum of SARS-CoV-2 positive patients and uninfected controls (Supplementary Table 1) for inflammatory mediators and the MC-specific proteases, chymase, $\beta$-tryptase, and CPA3. Consistent with previous reports, serum from SARS-CoV-2 patients had significantly higher levels of inflammatory mediators compared to uninfected controls, including CCL2, CCL3, CCL4, IP-10, IL-6, IL-8, VEGF, TNF, and IFN- $\gamma$ (Figure 1A and Supplementary Figure 1A). We also found significantly elevated levels of chymase, $\beta$-tryptase, and CPA3 in SARS-CoV2 patient serum, strongly suggesting systemic MC activation (Figure 1B). Consistent with increased MC activation, SARSCoV-2 patient serum also had significantly increased levels of catalytically active, mature tryptase compared to serum from uninfected donors (Figure 1C). To gain additional insight into the association between inflammation and MC activation in SARS-CoV-2 patients, we correlated inflammatory cytokines and chemokines with MC-derived proteases. Protease levels positively correlated with levels of many inflammatory cytokines associated with COVID-19 disease severity, including IP-10, CCL2, and CCL4 (Figures 1D,E). Interestingly, the serum levels of MC-derived proteases showed little concordance (Figure 1D).
The significant elevation in MC proteases but lack of correlation could represent activation of different MC populations. These data demonstrate that serum levels of MC-specific proteases are elevated in a small cohort of SARS-CoV-2 patients with generalized inflammation and suggest $\mathrm{MC}$ activation is a feature of COVID-19 pathogenesis.

\section{Protease and Eosinophil Granule Genes Are Increased in COVID-19 Patient Lungs}

Following the characterization of MC-specific proteases in SARSCoV-2 patient serum, we next explored the expression of these mediators in lung tissues obtained from post-mortem COVID19 patients and control tissues from uninfected patients using publicly available RNA-seq datasets. Due to the small number of patients within each published study, we leveraged three different bulk RNA-seq datasets to generate a combined dataset with ten COVID-19 patient lungs and three lung tissue samples from uninfected individuals $(36,37)$. Transcriptional profiling of these samples revealed 1741 differentially expressed genes between infected lungs and controls (Supplementary Figure 1B).

The differential expression analysis identified elevated expression of individual inflammatory cytokines and chemokines in the lungs of COVID-19 patients, including CCL2 and IP-10 (Figure 2). Consistent with the serum findings from SARS-CoV2 patients, the MC protease genes TPSB2 and TPSAB1 which encode for $\alpha$ - and $\beta$-tryptase, respectively, were significantly elevated in lungs from COVID-19 patients, suggesting increased activation of lung MCs in COVID-19 inflammation (Figure 2). Interestingly, other MC-specific genes, such as SIGLEC6, HDC, KIT, and others were not increased in COVID-19 lung tissue. In addition, the MC-derived protease genes, CPA3 and CMA1 (chymase), were not increased in COVID-19 patient lung tissue, consistent with the tissue-specific protease expression profile found in lung MCs (Figure 2).

Given reports of eosinopenia in COVID-19 patients $(38,39)$, we were surprised to find that multiple eosinophil-associated granule genes were significantly upregulated in COVID-19 patient lung tissue, for example CLC (Galectin-10) and RNASE2 (EDN) (Figure 2). In addition, CCL11 (eotaxin-1), a major eosinophil chemokine, was significantly increased in COVID19 lung patient tissue. To evaluate if these eosinophil-related proteins were also elevated in serum, we measured EDN and CCL11 in SARS-CoV-2 patient and uninfected donor serum. Consistent with the gene expression analysis, EDN and CCL11 were significantly elevated in SARS-CoV-2 patient serum (Supplementary Figure 1C). Collectively, these data show that MC-derived proteases and eosinophil-associated mediators are increased in COVID-19 lungs and/or serum and further suggest these cells could be components of COVID-19 inflammation.

\section{TLR Stimulation of MCs Induces Activation That Resembles Features of SARS-CoV-2 Inflammation}

We next investigated potential mechanisms of $\mathrm{MC}$ and eosinophil activation during SARS-CoV-2 inflammation. 


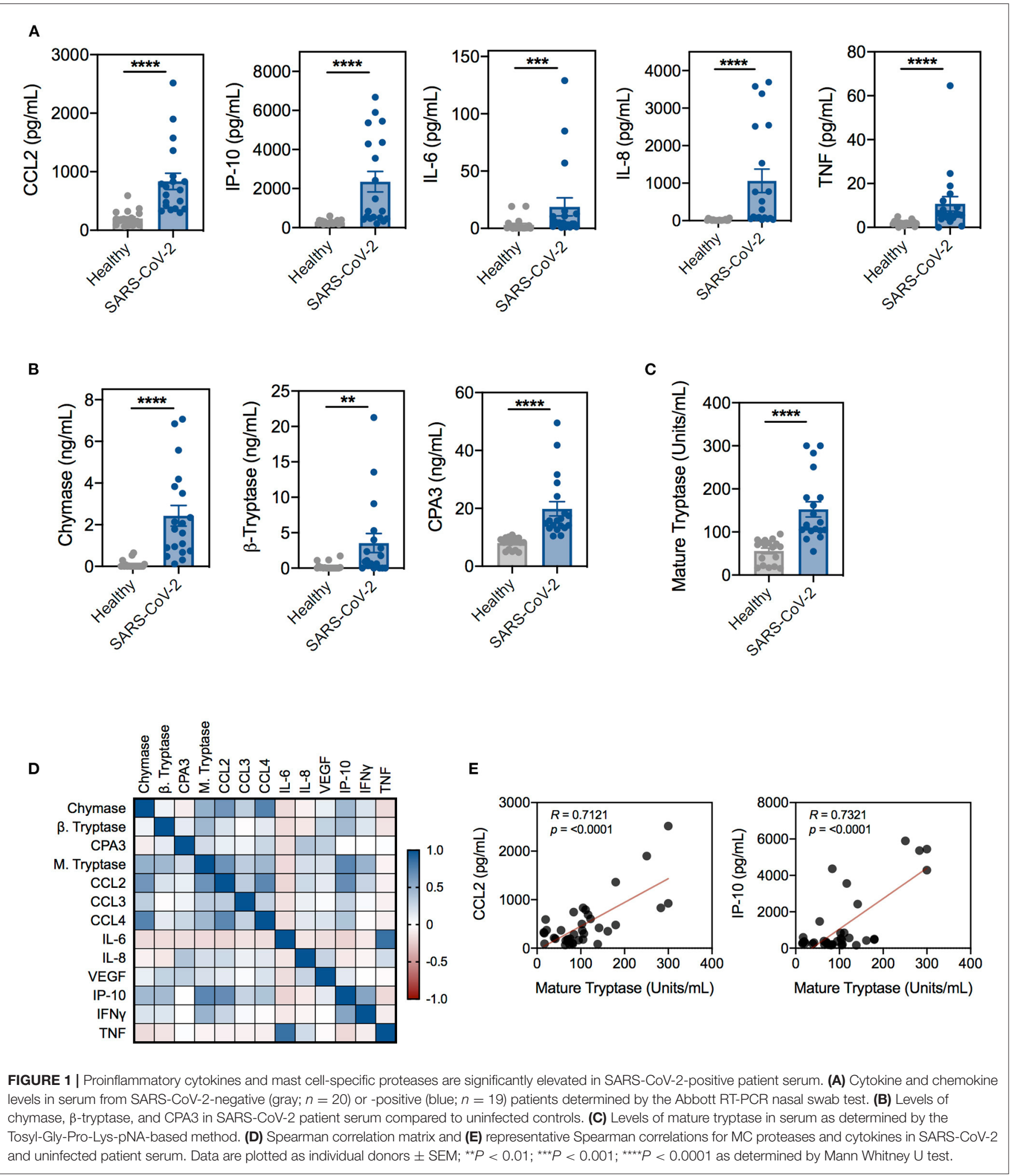

Peripheral blood-derived human MCs and purified blood eosinophils from healthy donors had minimal expression of the cell entry receptor for SARS-CoV-2, angiotensin-converting enzyme 2 (ACE2) relative to infection-competent Calu-3 cells (Supplementary Figure 2A) (40). Consistent with the expression on peripheral blood-derived MCs, FACS-sorted healthy donor 


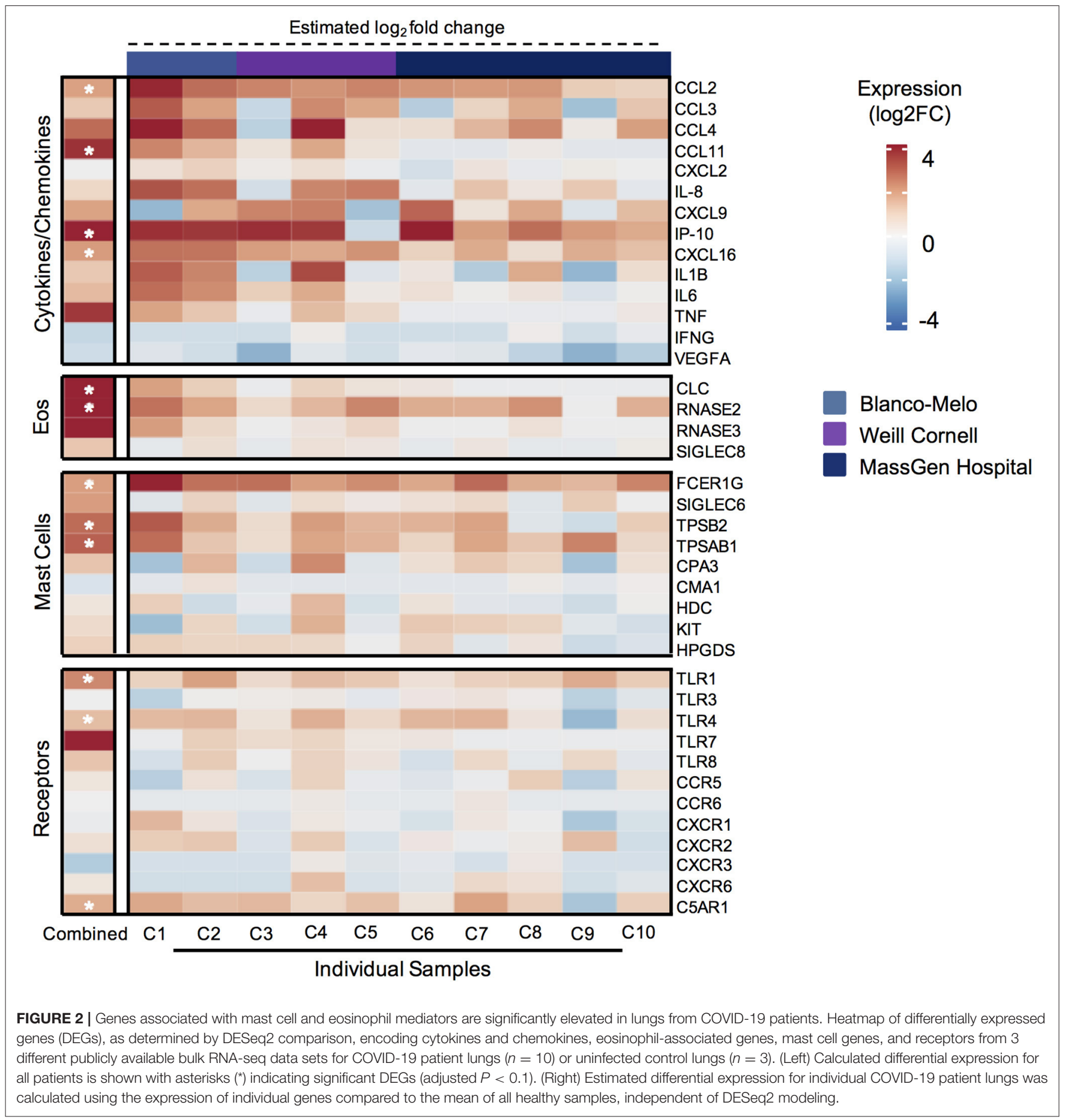

lung tissue MCs also displayed low expression of ACE2 (Supplementary Figures 2A,B). To determine if the engagement of TLRs by viral pathogens could mediate MC and eosinophil activation, we stimulated these cells with the synthetic analogs of ssRNA and dsRNA, R848 and poly (I:C), respectively. Both R848 and poly (I:C) induced significant $\mathrm{MC}$ activation as evidenced by increased cytokine release, including IL-8, CCL3, and CCL4 (Supplementary Figure 3A). In addition, these viral RNA analogs induced release of chymase and active mature tryptase (Supplementary Figure 3B). In contrast to MCs, only R848 induced eosinophil activation as evidenced by increased surface expression of CD69 and cytokine and chemokine production (Supplementary Figures 3C,D). However, we did not detect increased levels of EDN from TLR stimulated human blood eosinophils, suggesting eosinophil granule release is not directly induced by viral TLR stimulation (data not shown). These data 
demonstrate that TLR3/7/8 stimulation induces activation of MCs and to a lesser extent, eosinophils that resemble these cell-specific mediator profiles seen in SARS-CoV-2 serum and COVID-19 lung samples.

\section{Siglec-8 mAb Treatment in vivo Suppresses TLR-Driven Inflammation Induced by Poly (I:C)}

Next, we wanted to examine the role of MCs and eosinophils in vivo in a TLR-specific viral inflammation mouse model where the anti-inflammatory activity of a Siglec- $8 \mathrm{mAb}$ (anti-S8) could be evaluated. To this end, we intratracheally instilled poly (I:C) to stimulate TLR3 and RIG-I-MDA5 pathways in vivo into Siglec8 transgenic mice that selectively express functional Siglec- 8 on mouse MCs and eosinophils (Figure 3A) (25). Administration of poly (I:C) in vivo has been used to mimic acute respiratory exacerbations triggered by viral infections $(41,42)$.

Intratracheal administration of poly (I:C) induced robust and significant airway inflammation as evidenced by infiltration of immune cells into the BAL fluid, including total leukocytes, monocytes, and neutrophils compared to vehicle control (Figures 3B,C and Supplementary Figure 4A). In addition, poly (I:C) increased blood monocytes and neutrophils (Figure 3D). One dose of anti-S8 significantly suppressed TLR-mediated infiltration of immune cells into the airway and expansion in the periphery (Figures 3B-D). Consistent with the robust immune cell infiltration into the airway, poly (I:C) administration increased cytokines and chemokines in the BAL fluid and serum (Figures 3E,F). Treatment with anti-S8 significantly reduced local and systemic cytokine and chemokine elevation induced by poly (I:C) (Figures 3E,F). These data suggest that intratracheal poly (I:C) administration results in dysregulated secretion of inflammatory cytokines and immune cell infiltration that is suppressed with administration of a Siglec- $8 \mathrm{mAb}$.

\section{Poly (I:C)-Driven Inflammation Is Associated With MC and Eosinophil Activation That Is Suppressed With a Siglec-8 mAb}

Next, we assessed if the decrease in poly (I:C)-mediated inflammation in mice treated with anti-S8 was associated with reduced $\mathrm{MC}$ and eosinophil activity. Interestingly, poly (I:C) administration significantly reduced eosinophil numbers in the BAL fluid and peripheral blood compared to control mice (Figure 4A). In contrast, eosinophil peroxidase (EPX) and eosinophil cationic protein (ECP) levels in the BAL fluid and serum were significantly increased in mice that received poly (I:C), suggesting TLR-driven inflammation induces activation, degranulation and subsequent reduction of eosinophils (Figures 4A-C). In support of eosinophil activation, CCL11 levels in the BAL fluid were significantly increased in mice administered poly (I:C) (Supplementary Figure 4B). Consistent with its known eosinophil depleting activity, anti-S8-treated mice had significantly reduced eosinophils in the BAL fluid and blood and decreased levels of EPX and ECP compared to isotype control mAb-treated mice administered poly (I:C) (Figures 4A-C).

To evaluate MC activation, we quantified levels of the MCspecific mediators, mast cell proteases -1 and -4 (MCPT- 1 and MCPT-4) in ex vivo cultured lung tissue and serum in poly (I:C)driven inflammation. Among the murine chymases, MCPT-4 is most likely the functional counterpart of human chymase because it has similar substrate specificity and proteoglycanbinding properties (43). Intratracheal poly (I:C) administration markedly increased MCPT-4, but not MCPT-1 levels in the lung and serum, indicative of MC activation (Figure 4D, data not shown). Anti-S8 treatment significantly reduced lung and serum MCPT-4 levels compared to isotype control mAb-treated mice, consistent with MC inhibition (Figure 4D). Lastly, to determine if $\mathrm{MC}$ protease levels were associated with proinflammatory cytokines in our poly (I:C) inflammation model, we correlated serum MCPT-4 levels with CCL2, IP-10, and IL-6. As was seen in human serum $(44,45)$, MC protease levels significantly correlated with many proinflammatory cytokines associated with poly (I:C) inflammation (Figure 4E). These data demonstrate that TLRmediated inflammation driven by synthetic viral RNA induces mouse $\mathrm{MC}$ and eosinophil activation that is suppressed with an anti-Siglec- $8 \mathrm{mAb}$.

\section{Siglec-8 mAb Treatment Reduces Disease Severity and Airway Inflammation Induced by RSV Infection}

To determine if an anti-Siglec- $8 \mathrm{mAb}$ could also suppress inflammation driven by human respiratory viruses, we infected Siglec- 8 transgenic mice with RSV (Figure 5A). RSV is a major viral pathogen of infants and adults and has been shown to activate MCs and eosinophils through PRRs (46, 47). Mice infected with RSV demonstrated significant weight loss on days $1-3$ post infection (dpi) compared to sham-treated mice (Figure 5B). Anti-S8 treatment significantly improved RSV infection-associated weight loss. Infection with RSV also induced significant immune cell airway infiltration, including increased numbers of lymphocytes, monocytes, and eosinophils in the BAL fluid (Figure 5C). Consistent with reduced weight loss seen in anti-S8-treated mice, airway inflammation was significantly decreased (Figure 5C). However, RSV viral titers were similar in isotype control- and anti-S8-treated mice (Figure 5D), suggesting that the reduction in cellular inflammation did not compromise protection against RSV infection. These data demonstrate that anti-Siglec-8 mAb-treatment can suppress RSV-induced lung inflammatory cell accumulation without worsening viral infection.

\section{DISCUSSION}

A hyperinflammatory response mediated by dysregulated immune cells is considered to play a detrimental role in the progression of COVID-19. While the majority of studies have implicated monocytes and macrophages as primary drivers of aberrant inflammation, very few studies have examined the role of other innate immune cells in COVID-19 inflammation (48). 


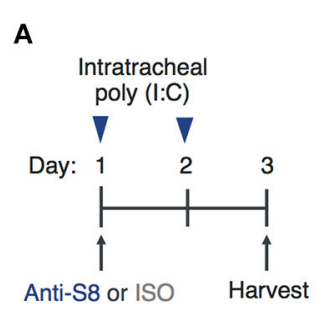

C

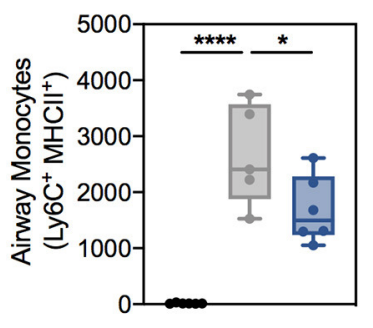

E

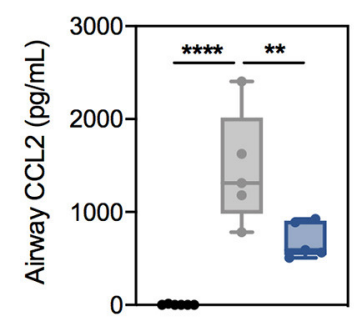

$\mathbf{F}$

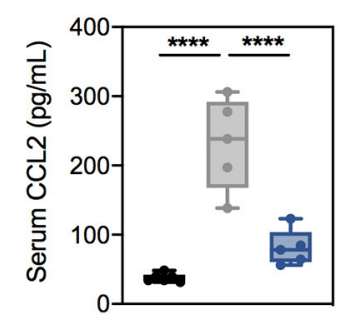

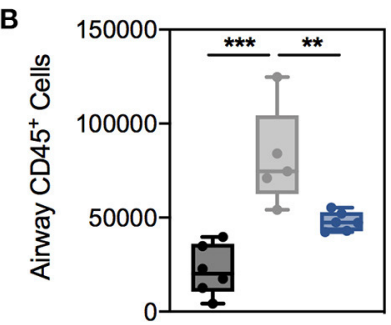
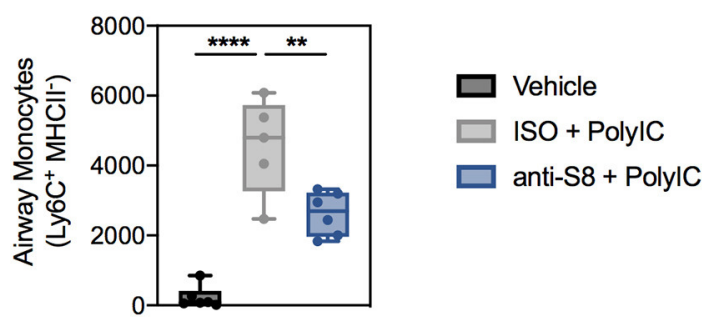

D
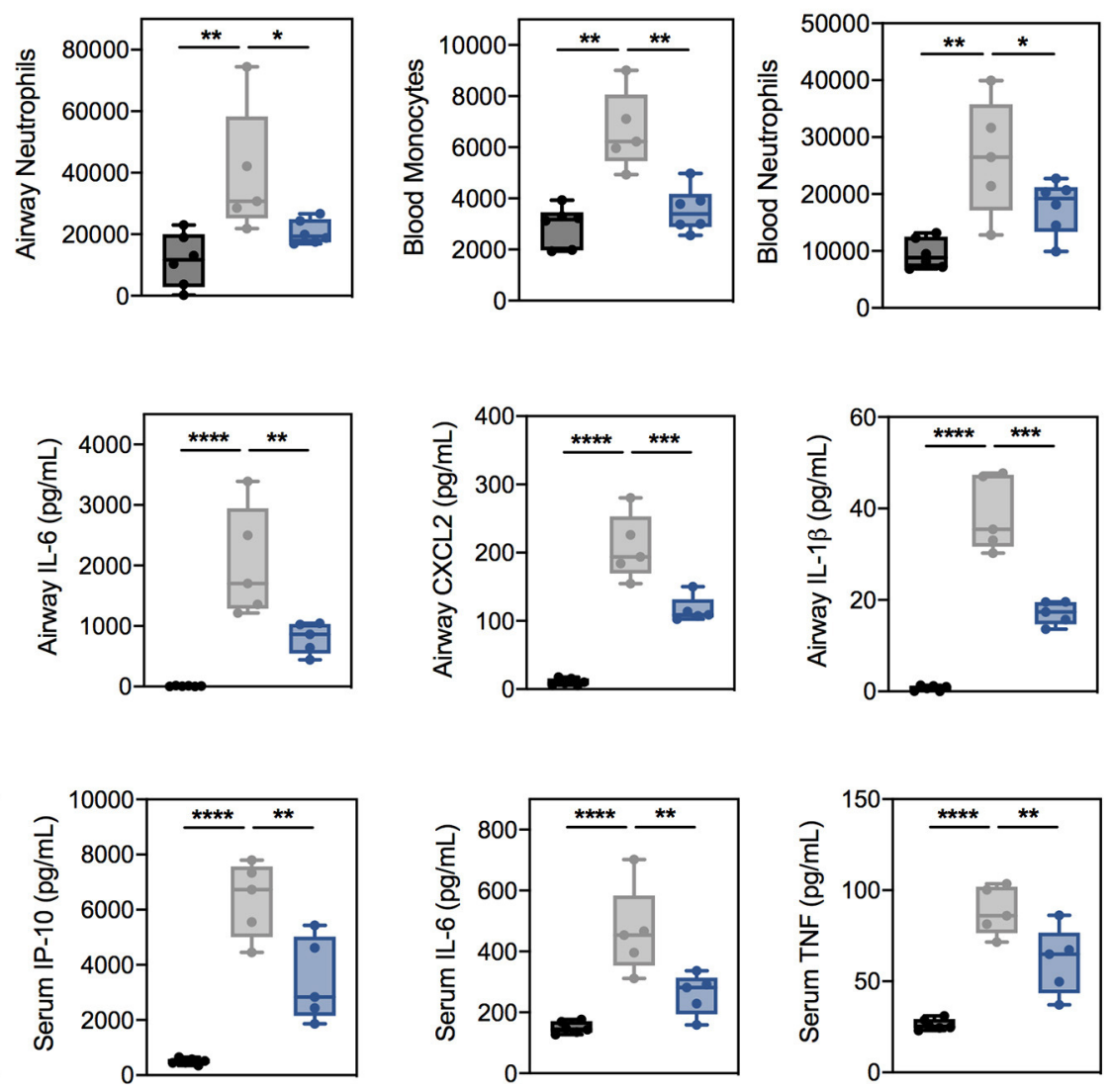

FIGURE 3 | Siglec-8 mAb treatment suppresses TLR-driven inflammation induced by poly (I:C) administration. (A) Schematic of poly (l:C)-mediated viral inflammation model. (B,C) Total leukocytes, $\mathrm{MHCll}^{-}$monocytes, $\mathrm{MHCl}^{+}$monocytes, and neutrophils in the BAL fluid or (D) monocytes and neutrophils in the blood of vehicle (black), ISO + poly (I:C) (gray), or anti-S8 + poly (I:C) (blue) treated mice as determined by flow cytometry. Levels of (E) CCL2, IL-6, CXCL2, and IL-1b in BAL fluid or (F) CCL2, IP-10, IL-6, and TNF in the serum of vehicle (black), ISO + poly (I:C) (gray), or anti-S8 + poly (I:C) (blue) treated mice. Data are plotted as mean \pm SEM (5-6 mice/group) and are representative of at least 2 experiments. ${ }^{\star} P<0.05$; ${ }^{\star \star} P<0.01$; ${ }^{\star \star \star} P<0.001$; ${ }^{\star \star \star \star} P<0.0001$ by one-way ANOVA with Tukey's

multiple-comparisons test. BAL, bronchoalveolar lavage; ISO, isotype control.

In the current study, we evaluated serum from SARS-CoV2 positive patients and publicly available RNA-seq data from diseased patients to examine the presence of MCs in COVID-19 inflammation. We found significant evidence of MC activation in the serum of SARS-CoV-2 patients and COVID-19 patient lung tissue by examining the levels and expression of MCspecific proteases. The levels of these proteases also significantly correlated with inflammatory cytokines implicated in COVID19 disease severity, including CCL2 and IP-10, suggesting MC activity could be associated with a heightened immune response. In support of this, post-mortem lung biopsies of COVID-19 patients showed increased density of perivascular and septal MCs compared to controls (30). Recent evidence also indicates that MCs can play a role in initiating hyperinflammation or cytokine storm in multiple disease settings $(18,49,50)$. Our findings also suggest systemic MC activation is associated with COVID-19 inflammation since we saw signatures of elevated proteases thought to be released from different MC populations. 

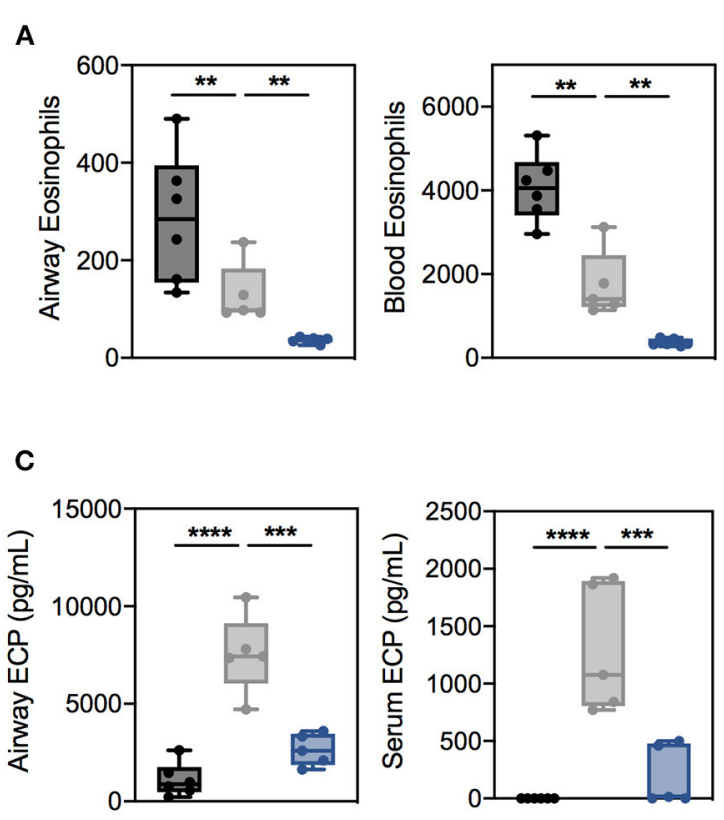

B
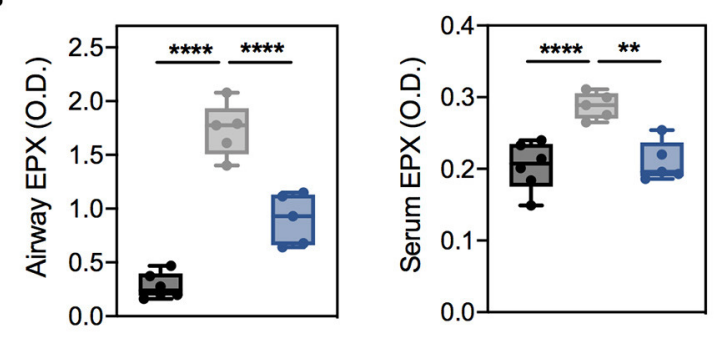

D

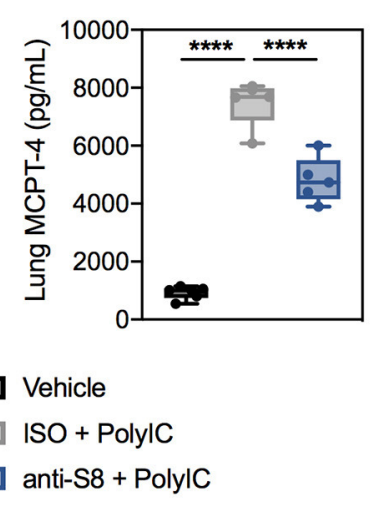

0

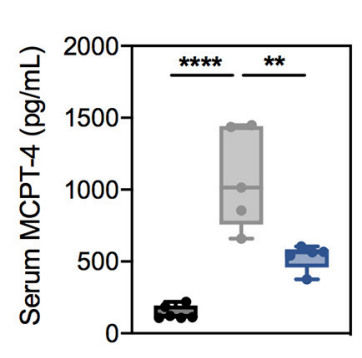

\section{.}
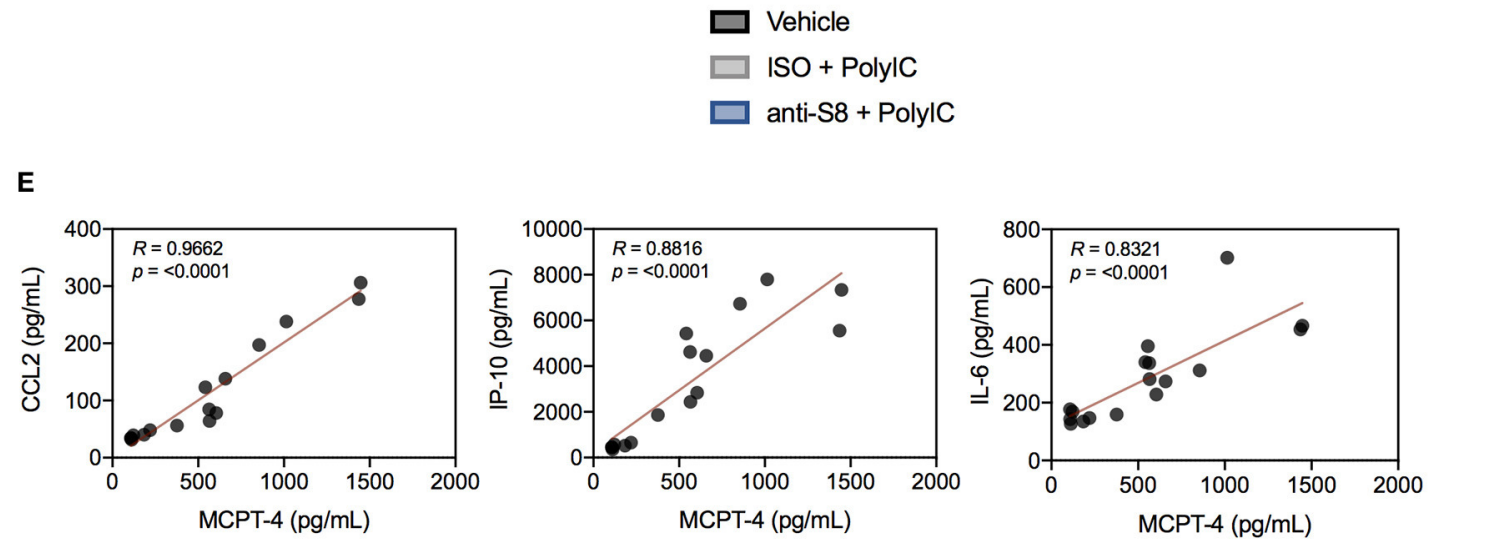

FIGURE 4 | Poly (I:C)-driven inflammation is associated with aberrant MC and eosinophil activation that is suppressed with a Siglec-8 mAb. (A) Eosinophils in the BAL fluid and blood, (B) EPX levels in BAL fluid (4-fold dilution) or serum (100-fold dilution) as measured by ELISA-determined optical density (O.D.), and (C) ECP levels in BAL fluid or serum from vehicle (black), ISO + poly (I:C) (gray), or anti-S8 + poly (I:C) (blue) treated mice. (D) Levels of MCPT-4 from overnight ex vivo lung cultures and serum in vehicle (black), ISO + poly (I:C) (gray), or anti-S8 + poly (I:C) (blue) treated mice. (E) Spearman correlations for serum MCPT-4, CCL2, IP-10, and IL-6 in vehicle, ISO + poly (I:C), or anti-S8 + poly (I:C) mice. Data are plotted as mean \pm SEM (5-6 mice/group) and are representative of at least 2 experiments. ${ }^{\star \star} P<0.01$; ${ }^{* * *} P<0.001 ;{ }^{* \star *} P<0.0001$ by one-way ANOVA with Tukey's multiple-comparisons test. BAL, bronchoalveolar lavage; ISO, isotype control; MCPT-4; mast cell protease-4.

Indeed, multiorgan clinical manifestations have been noted in some COVID-19 patients and our data supports evaluation of $\mathrm{MC}$ activation in these patients in the future (51).

MCs are key effector cells that regulate acute and chronic inflammatory responses through the release of preformed and de novo synthesized mediators in response to a diverse array of activating stimuli. Of these mediators, the MC-derived proteases, $\beta$-tryptase, chymase, and CPA- 3 are considered to be specific for MCs and indicative of the activation state of MCs $(52,53)$. These proteases induce broad biological responses, including proliferation and contraction of smooth muscle cells, cleavage of angiotensin (ang)-I, and activation of peptides and enzyme precursors, such as matrix metalloproteinases, TGF- $\beta$, kallikrein (which produces bradykinin) and IL-18 (43). As such, amplified release of these enzymes is implicated in the pathogenesis of numerous diseases, as well as detrimental effects in viral infections $(20,54)$. Increased levels of chymase and tryptase are seen in severe DENV and JEV infection and contribute to the development of vasculopathy and thrombocytopenia $(20,55)$. Evidence of systemic vasculopathy, angiogenesis, and coagulopathy has also been found in COVID-19 patients and is suspected to contribute to pathogenesis (56). While our data 
A

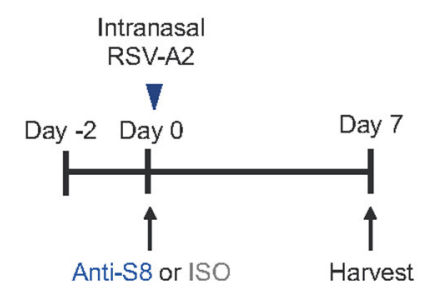

B

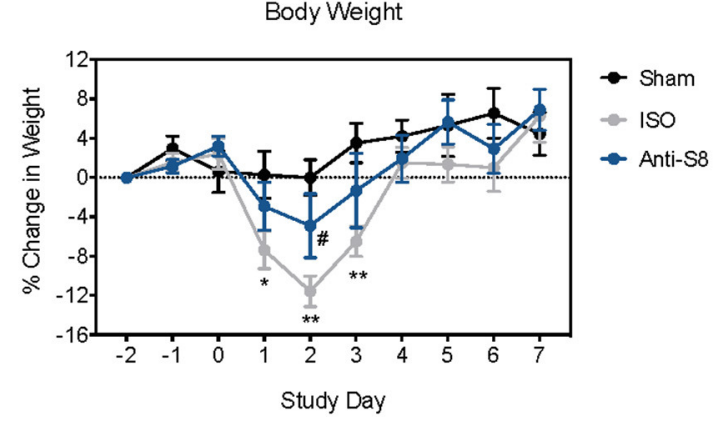

C

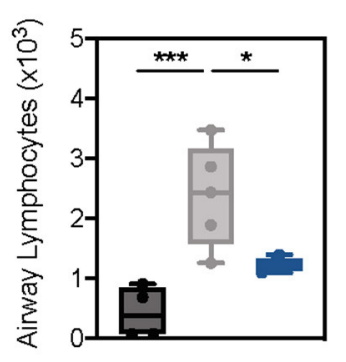

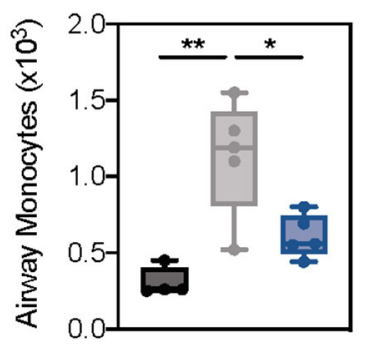

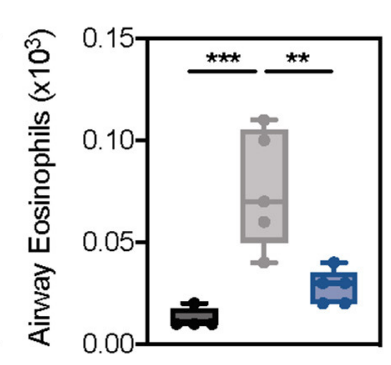

D

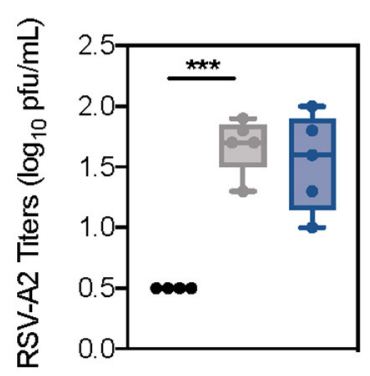

Sham
ISO + RSV
anti-S8 + RSV

FIGURE 5 | Siglec-8 mAb treatment reduces RSV-mediated airway inflammation without modulating viral titer. (A) Schematic of RSV-driven infection model in Siglec-8 transgenic mice. (B) Percent change in body weight from day-2 to day 7 in sham (black), ISO + RSV (gray), or anti-S8 + RSV (blue) treated mice. (C) Numbers of airway lymphocytes, monocytes, and eosinophils or (D) viral titer in BAL fluid on day 7 post infection of sham (black), ISO + RSV (gray), or anti-S8 + RSV (blue) treated mice. Data are plotted as mean \pm SEM (4-5 mice/group). ${ }^{\star} P<0.05 ;{ }^{* \star} P<0.01$; ${ }^{\star \star \star} P<0.001$; \#P $<0.05$ (ISO vs. anti-S8, B) by one-way ANOVA with Tukey's multiple-comparisons test. BAL bronchoalveolar lavage, ISO isotype control.

do not directly compare vascular factors, elevated levels of chymase and tryptase have been directly linked to virus-induced vasculopathy and thrombotic microangiopathies (20).

Cellular entry through ACE2 is the dominant mechanism for SARS-CoV-2 $(40,57)$. However, studies have failed to directly link hyperinflammation with the expression profile of ACE2 on immune cells. In support of this, we detected low expression of ACE2 on MCs and eosinophils, compared to infection-permissive Calu-3 cells. Therefore, rather than direct infection, it is likely is that secondary activation of innate immune cells like MCs and eosinophils via PRRs contributes to the release of inflammatory cytokines. Indeed, innate sensors that recognize viral RNA, including TLR3/7/8, become overstimulated in response to SARS-CoV, and in vivo administration with viral RNA analogs induce barrier damage to the airways upon TLR activation $(42,58)$. We observed that TLR stimulation with synthetic viral RNA directly activated MCs in vitro and induced features of hyperinflammation in vivo, including systemic and local inflammatory cytokine production, infiltration of neutrophils and monocytes in the airway, and production of the MC-protease, MCPT- 4 .
Interestingly, our gene expression analysis of post-mortem COVID-19 patient lungs revealed that the eosinophil associated genes, CLC, RNASE2 (EDN), and CCL11 were some of the most upregulated genes in COVID-19 lung tissue. An elevation of these genes in lung tissue was striking given that eosinopenia has been a common finding in patients admitted with COVID$19(38,39)$. CCL11 has been shown to induce degranulation and EDN release from human eosinophils and contribute to cytolysis of eosinophils in mice which could potentially explain the counterintuitive finding of eosinopenia and elevated secondary granules in COVID-19 patients (59-61). In support of this, mice exposed to synthetic viral RNA in vivo displayed blood and airway eosinopenia, increased levels of CCL11, and elevated expression of EPX and ECP, highly suggestive of activation. Nonetheless, the precise role of eosinophils in COVID-19 inflammation remains unclear, with reports of elevated and activated peripheral eosinophils in severe cases of COVID-19 (62-64). We found that human eosinophils directly responded to TLR7/8 stimulation using R848 by producing inflammatory cytokines, consistent with previous studies (65). Neither TLR3 nor TLR7/8 stimulation induced EDN release, suggesting 
alternative modes of activation are required to induce secretion of secondary granules from human eosinophils. Although EDN and CLC are most abundantly expressed in eosinophils, they have also been found in other leukocytes, albeit at much lower quantities, which may account for the increased expression seen in COVID-19 patient samples (66). While the role of EDN in COVID-19 has not been widely described, it has previously been associated with neuroinflammatory disease and neuronal cell death (40). It is therefore tempting to speculate that the elevated levels of EDN observed in COVID-19 patients may contribute to neurological manifestations found in some COVID-19 patients.

There are currently a limited number of effective therapeutic strategies to treat excessive inflammation associated with viral infections, such as COVID-19. Using a well-established model of poly (I:C)-induced lung inflammation, we examined the therapeutic potential of targeting MCs and eosinophils using a Siglec- $8 \mathrm{mAb}$. Anti-Siglec- $8 \mathrm{mAbs}$ have previously been shown to reduce immune cell infiltration, cytokine production, and tissue damage in chronic non-allergic airway models of chronic obstructive pulmonary disease and idiopathic pulmonary fibrosis (24). However, to date, the activity of an anti-Siglec$8 \mathrm{mAb}$ has not been evaluated in a model of TLR-mediated inflammation. Treatment with anti-S8 significantly suppressed immune cell infiltration, local and systemic inflammation, and levels of MC-derived proteases induced by synthetic viral RNA. Given that both MCs and eosinophils have been shown to play an important role in viral clearance with certain pathogens $(7,17,18)$, we also investigated the effects of an anti-Siglec$8 \mathrm{mAb}$ in a murine model of RSV infection. Anti-Siglec$8 \mathrm{mAb}$ treatment significantly decreased RSV-induced lung inflammation without hampering antiviral immunity, suggesting MC inhibition and eosinophil depletion via anti-Siglec- 8 is an attractive approach for suppressing viral inflammation without compromising host defense. Moreover, since over $80 \%$ of asthma exacerbation episodes are associated with viral infections (67), these data highlight the potential use of anti-Siglec- $8 \mathrm{mAbs}$ as an approach to treat virus-induced asthma exacerbations.

While ITIM-containing receptors, such as Siglec-8, are mainly thought to inhibit immunoreceptor tyrosine activation motifs (ITAM)-bearing receptors, these data and previously published findings suggest Siglec-8 can inhibit both ITAMand non-ITAM-bearing receptors on MCs, including FceRI, ST2 (IL-33 receptor), MRGPRX2, and TLR (22-24, 68). Similarly, murine Siglec-E can inhibit TLR activation in a MyD88-dependent manner by decreasing NF- $\kappa \mathrm{B}$ activation and the secretion of proinflammatory mediators (69). Future studies will focus on further characterizing the intracellular pathways induced by Siglec- 8 ligation that lead to inhibition of $\mathrm{MC}$ responses.

This study has several limitations. The cohort of individuals used for serum collection and RNA-seq analyses was small and lacked robust clinical information, including disease severity, comorbidities, and patient outcomes. Follow up studies are needed on a well-characterized patient population. In addition, the blood-derived MCs and eosinophils used in these studies may not completely reflect the function and phenotype of mature diseased human tissue lung MCs and eosinophils. Lastly, the use of viral RNA mimetics or RSV to study MC and eosinophil responses to SARS-CoV-2 may not fully recapitulate the complexity of COVID-19.

The data presented here suggest that systemic MC and eosinophil activation play a role in the inflammatory mechanisms underlying disease pathogenesis of COVID-19. Importantly, these data also demonstrate that targeting Siglec- 8 with an antibody reduces TLR-mediated inflammation and suggest that Siglec- 8 mAbs, such as liretelimab, could be used as a means of limiting immune cell infiltration and excessive inflammation in severe viral infection.

\section{DATA AVAILABILITY STATEMENT}

The raw data supporting the conclusions of this article will be made available by the authors, without undue reservation.

\section{ETHICS STATEMENT}

The studies involving human participants were reviewed and approved by Discovery Life Biosciences. The patients/participants provided their written informed consent to participate in this study. The animal study was reviewed and approved by Murigenics Inc., 941 Railroad Ave, Vallejo, CA 94592. Aragen Biosciences, 380 Woodview Ave, Morgan Hill, CA 95037.

\section{AUTHOR CONTRIBUTIONS}

SG, JS, MB, TL, EB, AX, AW, JL, and WK conducted the experiments. SG, JS, MB, and BY designed the experiments. KC and RM analyzed the RNA-seq data. BY, RS, and BB wrote the paper. All authors contributed to the article and approved the submitted version.

\section{FUNDING}

This research was funded by Allakos, Inc.

\section{ACKNOWLEDGMENTS}

Ingrid Koo, $\mathrm{PhD}$, provided editorial support and Dr. Bhupinder Singh for critical review.

\section{SUPPLEMENTARY MATERIAL}

The Supplementary Material for this article can be found online at: https://www.frontiersin.org/articles/10.3389/fimmu. 2021.650331/full\#supplementary-material 


\section{REFERENCES}

1. Chen N, Zhou M, Dong X, Qu J, Gong F, Han Y, et al. Epidemiological and clinical characteristics of 99 cases of 2019 novel coronavirus pneumonia in Wuhan, China: a descriptive study. Lancet. (2020) 395:507-13. doi: 10.1016/S0140-6736(20)30211-7

2. Huang C, Wang Y, Li X, Ren L, Zhao J, Hu Y, et al. Clinical features of patients infected with 2019 novel coronavirus in Wuhan, China. Lancet. (2020) 395:497-506. doi: 10.1016/S0140-6736(20)30183-5

3. Pedersen SF, Ho Y-C. SARS-CoV-2: a storm is raging. J Clin Invest. (2020) 130:2202-5. doi: 10.1172/JCI137647

4. Kox M, Waalders NJB, Kooistra EJ, Gerretsen J, Pickkers P. Cytokine levels in critically ill patients with COVID-19 and other conditions. JAMA. (2020) 324:1565-7. doi: 10.1001/jama.2020.17052

5. Giamarellos-Bourboulis EJ, Netea MG, Rovina N, Akinosoglou K, Antoniadou A, Antonakos N, et al. Complex immune dysregulation in COVID-19 patients with severe respiratory failure. Cell Host Microbe. (2020) 27:992-1000.e3. doi: 10.1016/j.chom.2020.04.009

6. Chen G, Wu D, Guo W, Cao Y, Huang D, Wang H, et al. Clinical and immunological features of severe and moderate coronavirus disease 2019. J Clin Invest. (2020) 130:2620-9. doi: 10.1172/JCI137244

7. Zhou Z, Ren L, Zhang L, Zhong J, Xiao Y, Jia Z, et al. heightened innate immune responses in the respiratory tract of COVID-19 patients. Cell Host Microbe. (2020) 27:883-90.e2. doi: 10.1016/j.chom.2020.04.017

8. Vabret N, Britton GJ, Gruber C, Hegde S, Kim J, Kuksin M, et al. Immunology of COVID-19: current state of the science. Immunity. (2020) 52:91041. doi: 10.1016/j.immuni.2020.05.002

9. Risitano AM, Mastellos DC, Huber-Lang M, Yancopoulou D, Garlanda C, Ciceri F, et al. Complement as a target in COVID-19? Nat Rev Immunol. (2020) 20:343-4. doi: 10.1038/s41577-020-0320-7

10. Tay MZ, Poh CM, Rénia L, MacAry PA, Ng LFP. The trinity of COVID19: immunity, inflammation and intervention. Nat Rev Immunol. (2020) 20:363-74. doi: 10.1038/s41577-020-0311-8

11. Galli SJ, Gaudenzio N, Tsai M. Mast cells in inflammation and disease: recent progress and ongoing concerns. Annu Rev Immunol. (2020) 38:4977. doi: 10.1146/annurev-immunol-071719-094903

12. St John AL, Abraham SN. Innate immunity and its regulation by mast cells. $J$ Immunol. (2013) 190:4458-63. doi: 10.4049/jimmunol.1203420

13. Wernersson S, Pejler G. Mast cell secretory granules: armed for battle. Nat Rev Immunol. (2014) 14:478-94. doi: 10.1038/nri3690

14. Galli SJ, Tsai M. IgE and mast cells in allergic disease. Nat Med. (2012) 18:693-704. doi: 10.1038/nm.2755

15. Mukai K, Tsai M, Saito H, Galli SJ. Mast cells as sources of cytokines, chemokines, and growth factors. Immunol Rev. (2018) 282:121-50. doi: 10.1111/imr.12634

16. Funk CD, Ardakani A. A novel strategy to mitigate the hyperinflammatory response to COVID-19 by targeting leukotrienes. Front Pharmacol. (2020) 11:1214. doi: 10.3389/fphar.2020.01214

17. Marshall JS, Portales-Cervantes L, Leong E. Mast cell responses to viruses and pathogen products. Int J Mol Sci. (2019) 20:4241. doi: 10.3390/ijms20174241

18. Graham AC, Temple RM, Obar JJ. Mast cells and influenza a virus: association with allergic responses and beyond. Front Immunol. (2015) 6:238. doi: 10.3389/fimmu.2015.00238

19. Aoki R, Kawamura T, Goshima F, Ogawa Y, Nakae S, Nakao A, et al. Mast cells play a key role in host defense against herpes simplex virus infection through TNF- $\alpha$ and IL-6 production. J Invest Dermatol. (2013) 133:21709. doi: $10.1038 /$ jid. 2013.150

20. St. John AL, Rathore APS, Raghavan B, Ng M-L, Abraham SN. Contributions of mast cells and vasoactive products, leukotrienes and chymase, to dengue virus-induced vascular leakage. Elife. (2013) 2:e00481. doi: 10.7554/eLife.00481.015

21. Bochner BS. "Siglec" ting the allergic response for therapeutic targeting. Glycobiology. (2016) 26:546-52. doi: 10.1093/glycob/cww024

22. Yokoi H, Choi O, Hubbard W, Lee H, Canning B, Lee H, et al. Inhibition of FcepsilonRI-dependent mediator release and calcium flux from human mast cells by sialic acid-binding immunoglobulin-like lectin 8 engagement. J Allergy Clin Immunol. (2008) 121:499-505. doi: 10.1016/j.jaci.2007.10.004
23. Youngblood BA, Brock EC, Leung J, Falahati R, Bryce PJ, Bright J, et al. AK002, a humanized sialic acid-binding immunoglobulin-like lectin- 8 antibody that induces antibody-dependent cell-mediated cytotoxicity against human eosinophils and inhibits mast cell-mediated anaphylaxis in mice. Int Arch Allergy Immunol. (2019) 180:91-102. doi: 10.1159/000501637

24. Schanin J, Gebremeskel S, Korver W, Falahati R, Butuci M, Haw TJ, et al. A monoclonal antibody to Siglec-8 suppresses non-allergic airway inflammation and inhibits IgE-independent mast cell activation. Mucosal Immunol. (2020). doi: 10.1038/s41385-020-00336-9

25. Youngblood BA, Brock EC, Leung J, Falahati R, Bochner BS, Rasmussen HS, et al. Siglec-8 antibody reduces eosinophils and mast cells in a transgenic mouse model of eosinophilic gastroenteritis. JCI Insight. (2019) 4:e126219. doi: 10.1172/jci.insight.126219

26. O'Sullivan JA, Chang AT, Youngblood BA, Bochner BS. Eosinophil and mast cell Siglecs: from biology to drug target. J Leukoc Biol. (2020) 108:7381. doi: 10.1002/JLB.2MR0120-352RR

27. Youngblood BA, Leung J, Falahati R, Williams J, Schanin J, Brock EC, et al. Discovery, function, and therapeutic targeting of Siglec-8. Cells. (2020) 10:19. doi: 10.3390/cells10010019

28. Theoharides TC, Conti P. COVID-19 and multisystem inflammatory syndrome, or is it mast cell activation syndrome? J Biol Reg Homeost Agents. (2020) 34:1633-6. doi: 10.23812/20-EDIT3

29. Conti P, Caraffa A, Tetè G, Gallenga CE, Ross R, Kritas SK, et al. Mast cells activated by SARS-CoV-2 release histamine which increases IL-1 levels causing cytokine storm and inflammatory reaction in COVID-19. J Biol Reg Homeost Agents. (2020) 34:1629-32. doi: 10.23812/20-2EDIT

30. Motta Junior J da S, Miggiolaro AFRDS, Nagashima S, de Paula CBV, Baena CP, Scharfstein J, et al. Mast cells in alveolar septa of COVID-19 patients: a pathogenic pathway that may link interstitial edema to immunothrombosis. Front Immunol. (2020) 11:574862. doi: 10.3389/fimmu.2020.574862

31. Hsieh JT, Rathore APS, Soundarajan G, St John AL. Japanese encephalitis virus neuropenetrance is driven by mast cell chymase. Nat Commun. (2019) 10:706. doi: 10.1038/s41467-019-08641-z

32. Abdin SM, Elgendy SM, Alyammahi SK, Alhamad DW, Omar HA. Tackling the cytokine storm in COVID-19, challenges and hopes. Life Sci. (2020) 257:118054. doi: 10.1016/j.lfs.2020.118054

33. Gigante A, Aquili A, Farinelli L, Caraffa A, Ronconi G, Enrica Gallenga C, et al. Sodium chromo-glycate and palmitoylethanolamide: a possible strategy to treat mast cell-induced lung inflammation in COVID-19. Med Hypotheses. (2020) 143:109856. doi: 10.1016/j.mehy.2020.109856

34. Kempuraj D, Selvakumar GP, Ahmed ME, Raikwar SP, Thangavel R, Khan A, et al. COVID-19, mast cells, cytokine storm, psychological stress, and neuroinflammation. Neurosci. (2020) 26:402-14. doi: 10.1177/1073858420941476

35. Theoharides TC. COVID-19, pulmonary mast cells, cytokine storms, and beneficial actions of luteolin. BioFactors. (2020) 46:306-8. doi: 10.1002/biof.1633

36. Blanco-Melo D, Nilsson-Payant BE, Liu W-C, Uhl S, Hoagland D, Møller $\mathrm{R}$, et al. Imbalanced host response to SARS-CoV-2 drives development of COVID-19. Cell. (2020) 181:1036-45.e9. doi: 10.1016/j.cell.2020.04.026

37. Desai N, Neyaz A, Szabolcs A, Shih AR, Chen JH, Thapar V, et al. Temporal and spatial heterogeneity of host response to SARS-CoV-2 pulmonary infection. Nat Commun. (2020) 11:6319. doi: 10.1038/s41467-020-20139-7

38. Du Y, Tu L, Zhu P, Mu M, Wang R, Yang P, et al. Clinical features of 85 fatal cases of COVID-19 from Wuhan. A retrospective observational study. Am J Respir Crit Care Med. (2020) 201:1372-9. doi: 10.1164/rccm.202003-0543OC

39. Zhang J-J, Dong X, Cao Y-Y, Yuan Y-D, Yang Y-B, Yan Y-Q, et al. Clinical characteristics of 140 patients infected with SARS-CoV-2 in Wuhan, China. Allergy. (2020) 75:1730-41. doi: 10.1111/all.14238

40. Hoffmann M, Kleine-Weber H, Schroeder S, Krüger N, Herrler T, Erichsen $\mathrm{S}$, et al. SARS-CoV-2 cell entry depends on ACE2 and TMPRSS2 and is blocked by a clinically proven protease inhibitor. Cell. (2020) 181:27180.e8. doi: 10.1016/j.cell.2020.02.052

41. Harris P, Sridhar S, Peng R, Phillips JE, Cohn RG, Burns L, et al. Double-stranded RNA induces molecular and inflammatory signatures that are directly relevant to COPD. Mucosal Immunol. (2013) 6:47484. doi: $10.1038 / \mathrm{mi} .2012 .86$ 
42. Broggi A, Ghosh S, Sposito B, Spreafico R, Balzarini F, Lo Cascio A, et al. Type III interferons disrupt the lung epithelial barrier upon viral recognition. Science. (2020) 369:706-12. doi: 10.1126/science.abc3545

43. Dell'Italia LJ, Collawn JF, Ferrario CM. Multifunctional role of chymase in acute and chronic tissue injury and remodeling. Circ Res. (2018) 122:31936. doi: 10.1161/CIRCRESAHA.117.310978

44. Herold T, Jurinovic V, Arnreich C, Lipworth BJ, Hellmuth JC, von BergweltBaildon M, et al. Elevated levels of IL-6 and CRP predict the need for mechanical ventilation in COVID-19. J Allergy Clin Immunol. (2020) 146:12836.e4. doi: 10.1016/j.jaci.2020.05.008

45. Laguna-Goya R, Utrero-Rico A, Talayero P, Lasa-Lazaro M, RamirezFernandez A, Naranjo L, et al. IL-6-based mortality risk model for hospitalized patients with COVID-19. J Allergy Clin Immunol. (2020) 146:799-807.e9. doi: 10.1016/j.jaci.2020.07.009

46. Al-Afif A, Alyazidi R, Oldford SA, Huang YY, King CA, Marr N, et al. Respiratory syncytial virus infection of primary human mast cells induces the selective production of type I interferons, CXCL10, and CCL4. J Allergy Clin Immunol. (2015) 136:1346-54.e1. doi: 10.1016/j.jaci.2015.01.042

47. Phipps S, Lam CE, Mahalingam S, Newhouse M, Ramirez R, Rosenberg $\mathrm{HF}$, et al. Eosinophils contribute to innate antiviral immunity and promote clearance of respiratory syncytial virus. Blood. (2007) 110:157886. doi: 10.1182/blood-2007-01-071340

48. Merad M, Martin JC. Pathological inflammation in patients with COVID19: a key role for monocytes and macrophages. Nat Rev Immunol. (2020) 20:355-62. doi: 10.1038/s41577-020-0331-4

49. Temple RM, Obar JJ. Influenza virus induces mast cell death independent of mast cell activation. J Immunol. (2017) 198 (1 Suppl.):203.13.

50. Valent P. KIT D816V and the cytokine storm in mastocytosis: production and role of interleukin-6. Haematologica. (2020) 105:5-6. doi: 10.3324/haematol.2019.234864

51. Zaim S, Chong JH, Sankaranarayanan V, Harky A. COVID19 and multiorgan response. Curr Probl Cardiol. (2020) 45:100618. doi: 10.1016/j.cpcardiol.2020.100618

52. Maun HR, Jackman JK, Choy DF, Loyet KM, Staton TL, Jia G, et al. An allosteric anti-tryptase antibody for the treatment of mast cell-mediated severe asthma. Cell. (2019) 179:417-31.e19. doi: 10.1016/j.cell.2019.09.009

53. Krystel-Whittemore M, Dileepan KN, Wood JG. Mast cell: a multi-functional master cell. Front Immunol. (2015) 6:620. doi: 10.3389/fimmu.2015.00620

54. Syenina A, Jagaraj CJ, Aman SAB, Sridharan A, St John AL. Dengue vascular leakage is augmented by mast cell degranulation mediated by immunoglobulin Fc $\gamma$ receptors. Elife. (2015) 4:e05291. doi: 10.7554/eLife.05291.008

55. Furuta T, Murao LA, Lan NTP, Huy NT, Huong VTQ, Thuy TT, et al. Association of mast cell-derived VEGF and proteases in Dengue shock syndrome. PLoS Negl Trop Dis. (2012) 6:e1505. doi: 10.1371/journal.pntd.0001505

56. Mondal R, Lahiri D, Deb S, Bandyopadhyay D, Shome G, Sarkar S, et al. COVID-19: are we dealing with a multisystem vasculopathy in disguise of a viral infection? J Thromb Thrombolysis. (2020) 50:56779. doi: 10.1007/s11239-020-02210-8

57. Shang J, Ye G, Shi K, Wan Y, Luo C, Aihara H, et al. Structural basis of receptor recognition by SARS-CoV-2. Nature. (2020) 581:2214. doi: 10.1038/s41586-020-2179-y

58. Birra D, Benucci M, Landolfi L, Merchionda A, Loi G, Amato P, et al. COVID 19: a clue from innate immunity. Immunol Res. (2020) 68:1618. doi: 10.1007/s12026-020-09137-5

59. Shamri R, Melo RCN, Young KM, Bivas-Benita M, Xenakis JJ, Spencer LA, et al. CCL11 elicits secretion of RNasesfrom mouse eosinophils and their cell-free granules. FASEB J Off Publ Fed Am Soc Exp Biol. (2012) 26:208493. doi: 10.1096/fj.11-200246

60. Melo RCN, Spencer LA, Perez SAC, Ghiran I, Dvorak AM, Weller PF. Human eosinophils secrete preformed, granule-stored interleukin-4 through distinct vesicular compartments. Traffic. (2005) 6:1047-57. doi: 10.1111/j.1600-0854.2005.00344.x

61. El-Shazly A, Masuyama K, Nakano K, Eura M, Samejima Y, Ishikawa T. Human eotaxin induces eosinophil-derived neurotoxin release from normal human eosinophils. Int Arch Allergy Immunol. (1998) 117 (Suppl.):558. doi: 10.1159/000053573

62. Lucas C, Wong P, Klein J, Castro TBR, Silva J, Sundaram M, et al. Longitudinal analyses reveal immunological misfiring in severe COVID-19. Nature. (2020) 584:463-9. doi: 10.1038/s41586-020-2588-y

63. Lippi G, Henry BM. Eosinophil count in severe coronavirus disease 2019 QJM. (2020) 113:511-2. doi: 10.1093/qjmed/hcaa137

64. Lindsley AW, Schwartz JT, Rothenberg ME. Eosinophil responses during COVID-19 infections and coronavirus vaccination. J Allergy Clin Immunol. (2020) 146:1-7. doi: 10.1016/j.jaci.2020.04.021

65. Nagase H, Okugawa S, Ota Y, Yamaguchi M, Tomizawa H, Matsushima $\mathrm{K}$, et al. Expression and function of Toll-like receptors in eosinophils: activation by Toll-like receptor 7 ligand. J Immunol. (2003) 171:397782. doi: 10.4049/jimmunol.171.8.3977

66. Lee JJ, Jacobsen EA, Ochkur SI, McGarry MP, Condjella RM, Doyle AD, et al. Human versus mouse eosinophils: "that which we call an eosinophil, by any other name would stain as red”. J Allergy Clin Immunol. (2012) 130:572-84. doi: 10.1016/j.jaci.2012.07.025

67. Busse WW, Lemanske RFJ, Gern JE. Role of viral respiratory infections in asthma and asthma exacerbations. Lancet. (2010) 376:826-34. doi: 10.1016/S0140-6736(10)61380-3

68. Gebremeskel S, Davis T, Wong A, Brock E, Leung J, Schanin J, et al. Siglec-8 antibody reduces substance P-induced inflammation by inhibiting MRGPRmediated mast cell activation. Allergy. (2020) 75:66-7.

69. Boyd CR, Orr SJ, Spence S, Burrows JF, Elliott J, Carroll HP, et al. Siglec-E is up-regulated and phosphorylated following lipopolysaccharide stimulation in order to limit TLR-driven cytokine production. J Immunol. (2009) 183:77039. doi: 10.4049/jimmunol.0902780

Conflict of Interest: JS, SG, MB, TL, EB, AX, AW, JL, WK, and BY are employees of Allakos and/or own stock options in Allakos. BB and RS did not perform any of the experiments but are paid consultants on the scientific advisory board of Allakos, Inc., and own stock in Allakos. BB and RS are coinventors on existing Siglec-8-related patents and thus may be entitled to a share of royalties received by Johns Hopkins University from Allakos, Inc. on the potential sales of such products. BB and RS are also cofounders of Allakos, which makes him subject to certain restrictions under university policy.

The remaining authors declare that the research was conducted in the absence of any commercial or financial relationships that could be construed as a potential conflict of interest.

Copyright (c) 2021 Gebremeskel, Schanin, Coyle, Butuci, Luu, Brock, Xu, Wong, Leung, Korver, Morin, Schleimer, Bochner and Youngblood. This is an open-access article distributed under the terms of the Creative Commons Attribution License (CC $B Y)$. The use, distribution or reproduction in other forums is permitted, provided the original author(s) and the copyright owner(s) are credited and that the original publication in this journal is cited, in accordance with accepted academic practice. No use, distribution or reproduction is permitted which does not comply with these terms. 